\title{
Elastic follow-up and relaxation of residual stresses
}

\author{
D J Smith ${ }^{1 *}$, J McFadden ${ }^{1}$, S Hadidimoud ${ }^{2}$, A J Smith ${ }^{1}$, A J Stormonth-Darling ${ }^{1}$, and A A Aziz ${ }^{1}$ \\ ${ }^{1}$ Solid Mechanics Group, Department of Mechanical Engineering, University of Bristol, Bristol, UK \\ ${ }^{2}$ Mechanical Engineering Department, Ferdowsi University of Mashhad, Iran
}

The manuscript was received on 21 May 2009 and was accepted after revision for publication on 5 October 2009.

DOI: 10.1243/09544062JMES1733

\begin{abstract}
A series of experiments were undertaken using a multiple bar assembly to measure elastic follow-up and relaxation of an initial residual stress. A test rig was designed to permit different levels of elastic follow-up to occur. The general features of the experimental results confirmed predictions provided by simple models. The most reliable measure of elastic followup was obtained by measuring the relaxation of the initial residual stress. The rate of relaxation of the residual stress is found to be proportional to the elastic follow-up factor.
\end{abstract}

Keywords: elastic follow-up, residual stress, experiments

\section{INTRODUCTION}

Residual stresses are created in many engineering components and usually as a consequence of the manufacturing process and final fabrication. Welding is a typical manufacturing process where, unless the component is subjected to post-weld heat treatment, the residual stress can attain a value close to or equal to the yield stress. Fabrication can also lead to additional locked-in stresses developed from fitting-up the different parts of an assembly [1].

The occurrence of residual stresses leads to a need to understand their role in structural integrity. For example, when a cracked structure, containing tensile residual stresses, is subjected to external loading, there is the potential for the structure to fail at a load lower than when the residual stresses are absent [2]. Alternatively, compressive residual stresses can be introduced to decrease the impact of applied stresses on fatigue and fracture. Examples include autofrettage of gun barrels and high-pressure piping [3], and cold-worked holes for aerospace applications [4].

In design procedures [5] and fracture assessment codes [6-8], stresses are classed according to whether they are primary or secondary. This is in order to avoid undertaking detailed numerical analysis. Secondary stresses are usually related with fixed displacements,

*Corresponding author: Solid Mechanics Group, Department of Mechanical Engineering, University of Bristol, Queen's Building, University Walk, Bristol, BS8 1TR, UK.

email:david.smith@bristol.ac.uk which do not contribute to plastic collapse, whereas, conversely, primary or fixed load stresses do play a part. Residual stresses arise because of incompatibility of strains and therefore are usually treated as secondary stresses. However, when residual stresses are seen as sufficiently long range and do not balance across a cracked section, these stresses are classed as primary. In practice, the boundary conditions on a structure can be any combination of primary and secondary stresses and understanding their interaction is difficult. Whether the residual stresses contribute to the primary stresses depends on two things: how plastic deformation or crack growth accommodates the original misfit and how the structure responds or follows up (elastically) when changes in relative stiffness occur as a consequence of plastic deformation or crack growth.

The implications of elastic follow-up were first considered by Robinson [9] in the relaxation of bolted joints because of creep. He showed that when the flange of the joint is rigid compared with the bolt, the elastic follow-up is insignificant. The force in the bolt reduces as creep strain in the bolt accumulates. When the flange is elastic, follow-up occurs since the ends of the bolt can move apart under the influence of the elastic flange. The effect of elastic follow-up on the creep of piping systems has been explored extensively [10-13], although in these articles, the magnitudes of elastic follow-up are defined differently from that suggested by Robinson. For example, Roche [12] presents elastic follow-up occurring when the least stressed parts act as a spring on the highly stressed part that is accumulating creep deformation. Although these previous articles are confined to creep at high temperatures, 
the phenomenon of elastic follow-up arises when the highly stressed parts exhibit any change in overall compliance whether through creep, plasticity, or crack growth.

Surprisingly, while there is a comprehensive body of research articles describing and analysing elastic follow-up and its consequences, there is only limited experimental evidence used to support the basic theories created around idealized structures such as bars in series or in parallel. Details of the idealized structures are presented later. Kobayashi et al. [14] undertook experiments on an aluminium alloy at $250{ }^{\circ} \mathrm{C}$ and obtained good agreement between measured and predicted effects of elastic follow-up during creep. Later work [15] undertook experiments on the effects of elastic follow-up under cyclic loading using stainless steel at $700^{\circ} \mathrm{C}$. Plasticity and creep were shown to make a contribution to the additional strain accumulated as a result of elastic follow-up. These latter two studies $[14,15]$ examined elastic follow-up for bars in series, whereas Kobatake et al. [16] examined a combined system of parallel and series bars and denoted elastic follow-up as being either global or local. The latter related to a stress concentration (e.g. a notch) and the global elastic follow-up associated with the bulk response of the structure. They tested stainless-steel bars at $550^{\circ} \mathrm{C}$ and correlated fatigue lives to the extent of elastic follow-up.

While it is recognized [17] that elastic follow-up (and shakedown) leads to the creation of a residual stress field, none of the earlier experimental studies examine directly the interaction of an initial residual stress and subsequent applied loading. Furthermore, as will be shown later, there is a direct relationship between elastic follow-up and relaxation of residual stresses just as there is a relationship between elastic followup and creation of residual stress [17]. The purpose of this article is to explain the design and development of experiments to measure elastic follow-up and, in particular, relaxation of initial residual stress. The article begins by describing simple models that summarize the basic concepts, with the models also being used to guide the design and development of a test rig. This is described together with the experimental procedures for testing the rig.

\section{ANALYSIS}

\subsection{Simple models}

Consider an assembly, as shown in Fig. 1, consisting of three bars, two outer bars (bar 3) and a central bar (with bars 1 and 2 in series), that are connected to upper and lower rigid plates. The assembly is subjected to a total load $F_{\mathrm{t}}$ with the corresponding displacement denoted by $\Delta$. It is assumed that the outer bars remain

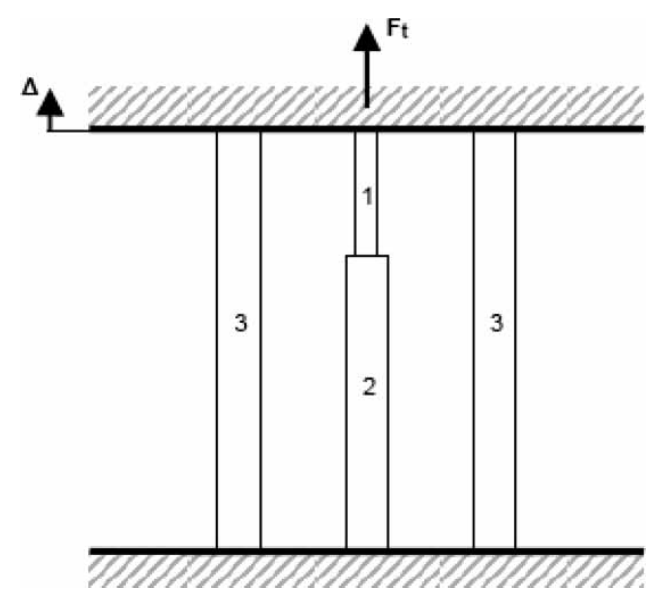

Fig. 1 Simple three-bar structure subjected to external loading

elastic and the central bar 1 undergoes perfectly plastic deformation when the stress exceeds the yield stress.

In the first instance, the central bar is considered as single entity, not in two sections as shown in Fig. 1, and the three-bar system is a parallel assembly. A typical elastic-perfectly-plastic response of the central bar is shown in Fig. 2. As the centre bar undergoes plastic deformation, the loading in the assembly is redistributed and the action of the elastic follow-up in the outer bars is to allow additional plastic deformation to occur in the central bar. If the loading on the assembly was simply displacement controlled, the total strain in the central bar would be $\varepsilon_{i}$ and the line R in Fig. 2 would be vertical. The slope of $R$ depends on the relative stiffness, $\alpha$ of the outer bars to the central bar

$$
\alpha=\frac{n K_{3}}{K_{1}}
$$

where $n$ is the number of outer bars, $K_{3}$ is the stiffness of a single outer bar, and $K_{1}$ is the stiffness of the central

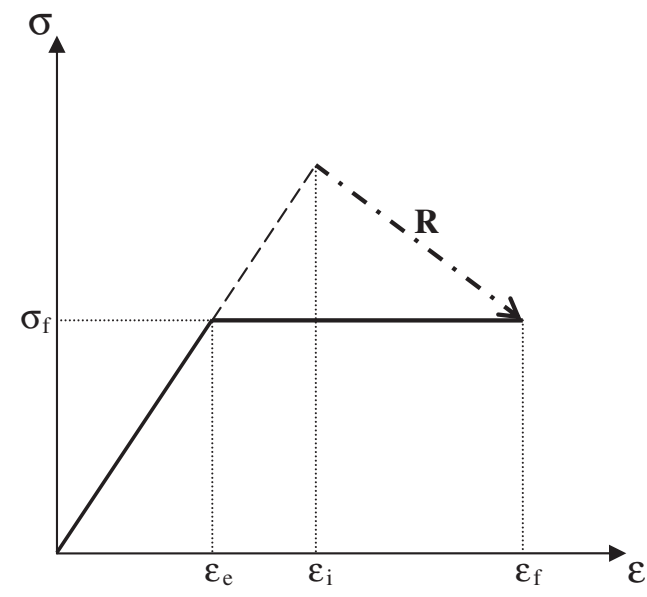

Fig. 2 Typical elastic-plastic response of centre bar in a three-bar assembly ( $\sigma=$ stress, $\varepsilon=$ strain) 
bar. For simple straight bars, the stiffness is given by

$$
K=\frac{E A}{L}
$$

where $E$ is Young's modulus, $A$ is the cross-sectional area, and $L$ is the length.

Following Kasahara [18], Kasahara et al. [19], and others [20], the elastic follow-up factor $Z$, for the central bar, is defined by

$$
Z=\frac{\varepsilon_{\mathrm{f}}-\varepsilon_{\mathrm{e}}}{\varepsilon_{\mathrm{i}}-\varepsilon_{\mathrm{e}}}
$$

where $\varepsilon_{\mathrm{f}}$ is the final total strain in the central bar achieved at the maximum load, $\varepsilon_{\mathrm{e}}$ is the equivalent elastic strain provided by the final stress $\sigma_{\mathrm{f}}$, and $\varepsilon_{\mathrm{i}}$ is the initial elastic strain that the central bar would achieve at the maximum load if it had not exhibited plastic deformation.

These quantities are illustrated in Fig. 2. The factor $Z$ has two extreme values, 1 and $\infty$. For displacementcontrolled conditions alone $Z=1$, and no additional strain is accumulated in the central bar. For loadcontrolled circumstances, the strains are unbounded and $Z$ tends to $\infty$.

The equations for the structural response of an assembly that combines series and parallel elements are given in Appendix 2. The elastic follow-up factor $Z_{\mathrm{p}}$, for the parallel assembly alone, is

$$
Z_{\mathrm{p}}=\frac{1+\alpha}{\alpha}
$$

Now consider the case of a series assembly with bars 1 and 2 in series and the cross-sectional area of bar 1 being less than bar 2. Assuming that the series assembly is subjected to a fixed displacement, it can be shown that the elastic follow-up factor $Z_{\mathrm{s}}$ is given by

$$
Z_{\mathrm{s}}=\frac{1+\beta}{\beta}
$$

where

$$
\beta=\frac{K_{2}}{K_{1}}
$$

Finally, for a combined parallel and series assembly shown in Fig. 1, the overall elastic follow-up factor $Z$ (derived in Appendix 2) is given by

$$
Z=Z_{\text {peff }} Z_{\mathrm{s}}=\left(\frac{1+\alpha_{\text {eff }}}{\alpha_{\text {eff }}}\right)\left(\frac{1+\beta}{\beta}\right)
$$

where $\alpha_{\text {eff }}$ is given by

$$
\alpha_{\text {eff }}=\frac{n K_{3}}{K_{\text {eff }}} \quad \text { and } \quad \frac{1}{K_{\text {eff }}}=\frac{1}{K_{2}}+\frac{1}{K_{1}}
$$

The elastic follow-up factor $Z$ is shown in Fig. 3 as a function of relative effective stiffness ratio. When the

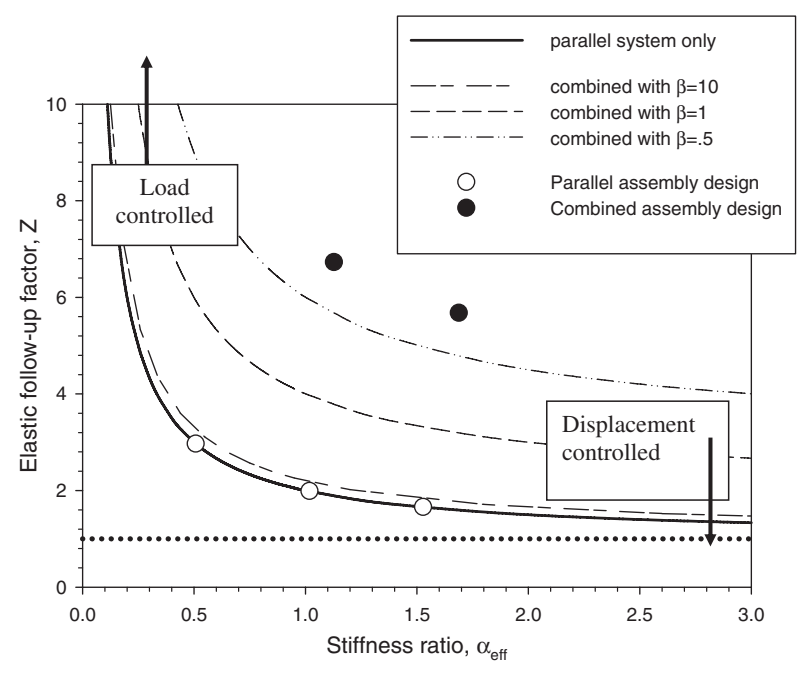

Fig.3 Extent of elastic follow-up as a function of effective relative stiffness

effective stiffness ratio is relatively low (less than about 0.2 , the elastic follow-up factor is in excess of 6 and the conditions are essentially load controlled. When the stiffness ratio is greater than $\sim 3$, the loading is in effect displacement controlled. It is also evident that for a combined parallel and series system, the addition of the series elements very rapidly increases $Z$ for a given value of the stiffness of the parallel system. This indicates that the central bar with the lower section area can be subjected effectively to load control even when the parallel system is providing a degree of displacement control.

\subsection{Response of assembly with an initial misfit}

A residual stress state can be created in the assembly through accommodating an initial misfit $\delta_{0}$ between the outer bars and the central bar. It can be shown that the residual force, $F_{1 \mathrm{R}}$, in bar 1 is given by

$$
F_{1 \mathrm{R}}=K_{1} \delta_{0}\left(\frac{\alpha_{\mathrm{eff}}}{1+\alpha_{\mathrm{eff}}}\right)
$$

This shows that the residual force (or stress) in the bar is a function of three parameters: the stiffness of the bar, the initial misfit, and the relative stiffness of the assembly. If, as a result of subsequent loading, any of these change, then on unloading the level of residual force will change. Further details of how changes to either the stiffness or misfit change the residual stress are explored by Aird et al. [21]. Importantly, if bar 1 undergoes sufficient plastic displacement equal to the initial misfit displacement, the initial stress reduces to zero.

To illustrate the behaviour of an assembly subjected to an initial misfit consider a simple three-bar assembly where there is a single central bar. It is assumed that for this purpose the assembly consists of bars of 
length $100 \mathrm{~mm}$, diameter $10 \mathrm{~mm}$, with the central bar made from steel with Young's modulus $210 \mathrm{GPa}$ and yield strength $365 \mathrm{MPa}$. It is assumed that the steel bar exhibits elastic-perfectly-plastic response. There are two outer bars manufactured from an aluminium alloy with Young's modulus $69 \mathrm{GPa}$.

It is assumed that an initial misfit of $0.1 \mathrm{~mm}$ is imposed on the central bar. The misfit conditions are shown in Fig. 4. Points A and B are the misfit stresses and strains in the central and one outer bar. On further external loading of the assembly, plasticity in the central bar begins and in doing so accommodates the original misfit. Eventually, when there is sufficient plastic strain to accommodate the entire initial misfit in the central bar, and then the assembly is unloaded, the residual stress returns to zero corresponding to points $C$ and $D$ in Fig. 4. The extent of the elastic followup provided by the assembly, represented by line $R$, is identical to the relaxation of the residual stress in the central bar, line $\mathrm{S}$.

Similar effects are observed for other assemblies irrespective of the relative stiffness of the assembly elements with the slopes of the lines $\mathrm{R}$ and $\mathrm{S}$ dictated by the relative stiffness. Consequently, the relaxation of the residual stress field in the assembly is identical to the elastic follow-up created by the assembly when the central bar undergoes plastic deformation. It is therefore possible to rewrite equation (3) as

$$
Z_{\mathrm{R}}=\frac{\varepsilon_{\mathrm{fR}}-\varepsilon_{\mathrm{eR}}}{\varepsilon_{0}-\varepsilon_{\mathrm{eR}}}
$$

where $Z_{\mathrm{R}}$ is the elastic follow-up related to the relaxation of the residual stress to a final relaxed strain, $\varepsilon_{\mathrm{fR}}$ in the central bar, $\varepsilon_{0}$ is the initial misfit strain, and $\varepsilon_{\mathrm{eR}}$ is the equivalent elastic strain associated with the relaxed residual stress.

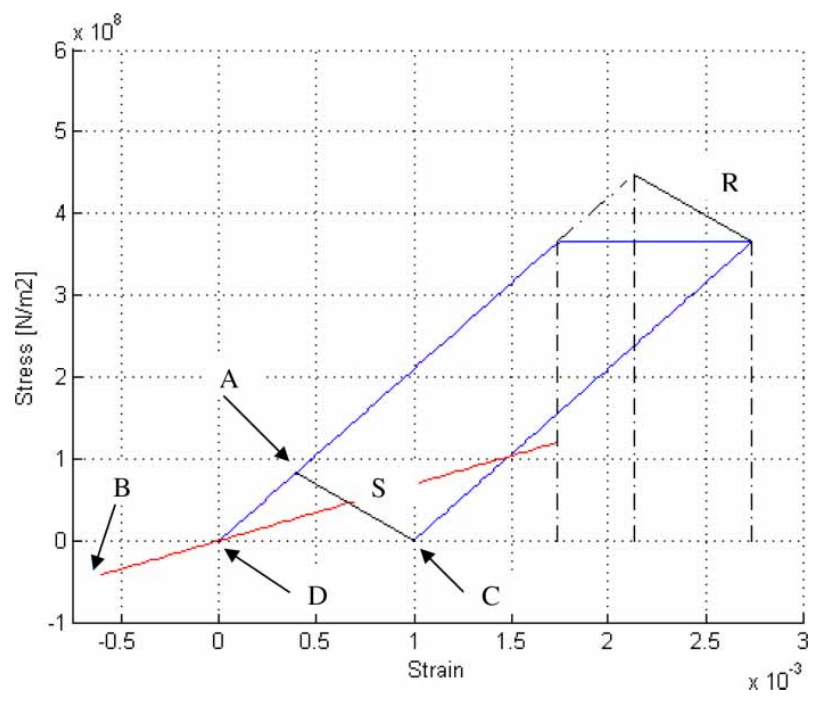

Fig. 4 Illustration of relaxation of initial residual force and misfit in a three-bar assembly
Finally, and although not shown in Fig. 4, if greater plastic deformation occurred in the central bar and the assembly is unloaded, it would be evident that a new compressive residual stress is created in the central bar in order to maintain compatibility [17].

These models were used to guide the development of an experimental rig. This is described in the next section.

\section{EXPERIMENTS}

\subsection{Experimental design}

An experimental rig was designed so that the mechanical behaviour of the components could be measured. Similar to the multiple bar concept described in the previous section, it was decided to replicate the concept as closely as possible and, principally, to ensure that uniform loads were applied to the elements. The design was constrained to be easy to assemble, have the ability to introduce known preloading, and then to be used in a standard servo-hydraulic test machine.

The overall arrangement of the rig is shown in Fig. 5 and consists of a central specimen, outer bars and high strength steel (EN24) upper and lower end sections. The overall length of the assembly was $490 \mathrm{~mm}$. In the first set of experiments, a standard carbon steel tensile test specimen [22] was used. In a second set of experiments, a carbon steel central bar, with a reduced centre section, was used, as illustrated in Fig. 6. The relative stiffness of the assembly was changed by introducing pairs of outer bars.

The outer bars were manufactured from an alu-

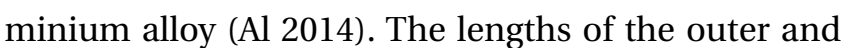
central bar were selected so that if the central bar experienced elastic-plastic deformation, the outer bars remained elastic.

The final design conditions for the parallel and combined assemblies are shown in Fig. 3 and also provided in Table 1, with largest elastic follow-up predicted to occur for the seven-bar combined parallel and series assembly.

\subsection{Test procedures}

Preliminary tests were conducted on steel and aluminium bars to determine precisely their elastic moduli and yield stresses. The tests were conducted in a servo-hydraulic machine together with an extensometer clipped onto the specimens. These tests revealed that $E$ for the steel and aluminium samples was 194 and $74 \mathrm{GPa}$, respectively. The yield stresses (corresponding to the first deviation from linearity) were 362 and $242 \mathrm{MPa}$ (with corresponding yield strains of 1865 and 3270 microstrain) for the steel and aluminium alloy bars, respectively. 


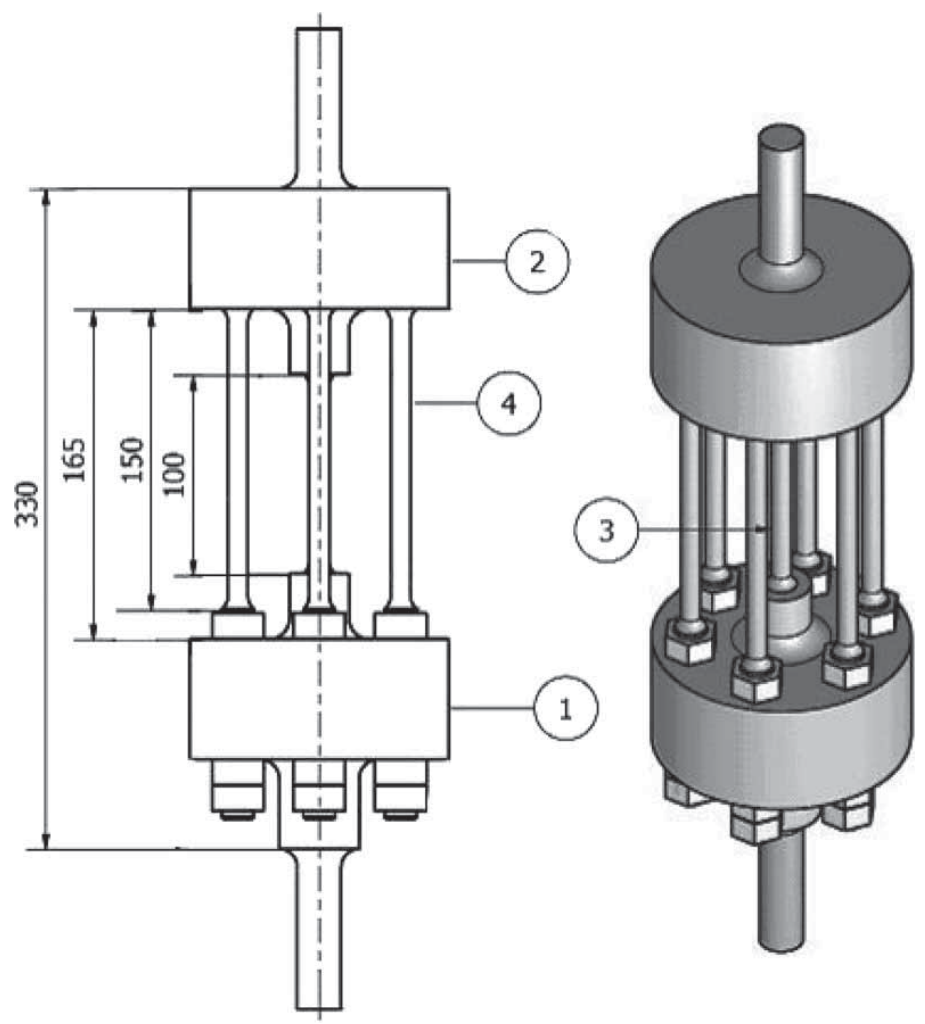

\begin{tabular}{|c|c|c|}
\hline Part No & Part & Material \\
\hline 1 & Lower End Section & EN24T Steel \\
\hline 2 & Upper End Section & EN24T Steel \\
\hline 3 & Central Specimen & Mild Steel \\
\hline 4 & Outer Bar & Aluminium \\
\hline
\end{tabular}

Fig. 5 Multiple bar assembly for experimental tests. All dimensions in mm

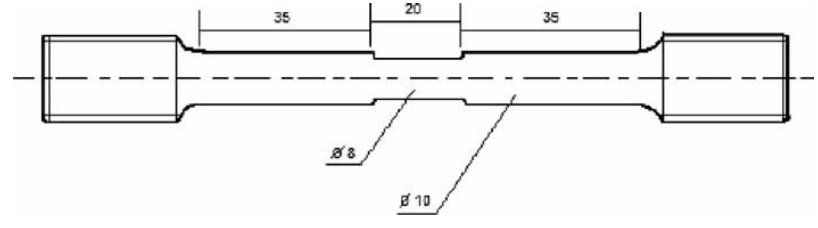

Fig. 6 Reduced section tensile specimen (all dimensions in $\mathrm{mm}$ )

Two series of tests were conducted using the assembly. The first set used standard central steel bars and was therefore treated as a simple parallel assembly. The second set used reduced section central bars thereby creating a combined parallel and series assembly. Each test used a new central bar. In the first series, the relative stiffness of the assembly was changed by using 2, 4, or 6 outer bars as shown in Table 1. Four tests were conducted in this series: two tests using two outer bars and two tests each with four and six outer bars. The second set of tests consisted of two tests, each using a reduced central bar and two and four outer bars. Strain gauges were installed on each of the bars prior to each test and assembly of the test rig.
Preloading of the centre bar was accomplished by inserting the assembly into the servo-hydraulic test machine and loading the assembly to introduce about 1000 microstrain to the central specimen. The outer bars were then locked into place and the load applied by the test machine removed. Subsequent loadings of the assembly were then undertaken under displacement control of $0.1 \mathrm{~mm} / \mathrm{min}$. Loading was continued until prescribed strains in the central bar were achieved. The external load was then released by displacing the test machine in the opposite direction, again at $0.1 \mathrm{~mm} / \mathrm{min}$. The load was allowed to relax to zero. Further load cycles were then applied until a point where it was necessary to prevent yielding occurring in the outer bars.

\section{RESULTS}

\subsection{Basic experimental characteristics}

Results for each test consisted of measured strains and applied loads and accurate measurement of the diameters and lengths of the bars. The initial elastic loading also permitted values of $E$ to be determined for each 
Table 1 Relative stiffness of assemblies and initial experimental results

\begin{tabular}{|c|c|c|c|c|c|c|}
\hline Assembly & $\begin{array}{l}\text { Idealized relative } \\
\text { stiffness, } \alpha \text { for } \\
\text { parallel system }\end{array}$ & $\begin{array}{l}\text { Idealized relative } \\
\text { stiffness, } \beta \text { for } \\
\text { series system }\end{array}$ & Predicted $Z$ & $\begin{array}{l}\text { Measured } \\
\text { relative } \\
\text { stiffness, } \alpha\end{array}$ & $\begin{array}{l}\text { Measured } Z \\
\text { Method } 1\end{array}$ & $\begin{array}{l}\text { Measured } Z_{\mathrm{R}} \\
\text { Method } 2\end{array}$ \\
\hline \multicolumn{7}{|c|}{ Series 1: parallel system tests } \\
\hline \multirow[t]{2}{*}{ Three-bar assembly } & 0.51 & - & 2.96 & 0.46 & 5.1 & 4.6 \\
\hline & & & & 0.47 & 4.6 & 4.3 \\
\hline Five-bar assembly & 1.02 & - & 1.98 & 1.0 & 3.4 & 3.0 \\
\hline Seven-bar assembly & 1.53 & - & 1.65 & 1.37 & 2.9 & 2.7 \\
\hline \multicolumn{7}{|c|}{ Series 2: combined parallel and series tests } \\
\hline Five-bar assembly & 1.13 & 0.39 & 6.72 & 1.14 & 5.5 & 5.1 \\
\hline Seven-bar assembly & 1.69 & 0.39 & 5.67 & 1.74 & 4.6 & 3.8 \\
\hline
\end{tabular}

bar and thereby allows the stiffness for each element of the assembly to be determined for all tests. The relative stiffness for each assembly was obtained from measured values of $E$ and using equations (2), (1), (6), and (8). Results are shown in Table 1.

Figure 7 illustrates typical results of load and strains for three- and seven-bar assemblies using a standard central bar. Results for the three-bar system are shown in Fig. 7(a). Initially, the system responded elastically but eventually the centre bar exhibited plastic
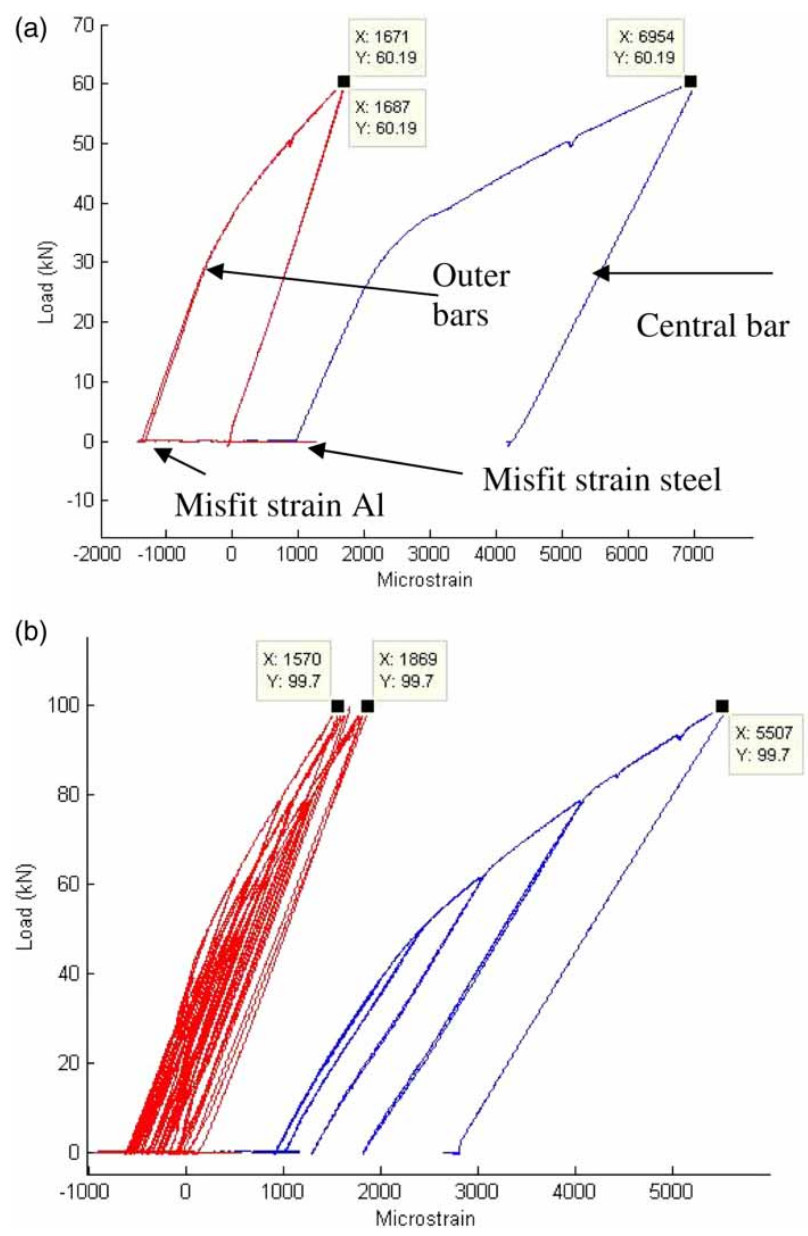

Fig. 7 Measured response of multibar structures subjected to external loading: (a) three-bar system and (b) seven-bar system deformation and subsequent non-linear behaviour was evident. There was no yielding in the outer bars because the final strain in the aluminium bars was well below the yield strain. Removal of the applied load resulted in complete elastic recovery of both the steel and aluminium bars.

Similar experimental results are shown for a sevenbar parallel assembly in Fig. 7(b). There was a small amount of non-symmetric loading in the outer bars. This equated to a bending stress of about $5 \mathrm{MPa}$ compared to the applied uniaxial stress on the aluminium bars of about $120 \mathrm{MPa}$. This level of non-symmetric strain was found to be a similar or less for all tests.

The stress and strain response of the central bar was determined from the results shown in Fig. 7. The information was then used to determine the relaxation of the initial stress created by misfit and the elastic follow-up factor for each test. The force $F_{\mathrm{A}}$ in one aluminium outer bar was determined from

$$
F_{\mathrm{A}}=\varepsilon_{\mathrm{A}} A_{\mathrm{A}} E_{\mathrm{A}}
$$

where $A_{\mathrm{A}}, \varepsilon_{\mathrm{A}}$, and $E_{\mathrm{A}}$ are the measured values of crosssectional area, strain, and Young's modulus for the aluminium outer bar. The average value of $F_{\mathrm{A}}$ was determined for each test.

The force $F_{\mathrm{S}}$ applied to the central steel bar was then determined using

$$
F_{\mathrm{S}}=F_{\mathrm{t}}-n F_{\mathrm{A}}
$$

where $n$ is the number of outer bars and $F_{\mathrm{t}}$ the total applied load by the test machine.

The stress in the central steel bar was determined using

$$
\sigma_{\mathrm{S}}=\frac{F_{\mathrm{S}}}{A_{\mathrm{S}}}
$$

where $A_{\mathrm{S}}$ is the measured cross-sectional area of the central steel bar.

For two tests conducted on three-bar assemblies, the calculated stress, as a function of measured strain, is shown in Fig. 8. Points A and B are the stress-strain pairs for the initial misfit condition, with point A being 

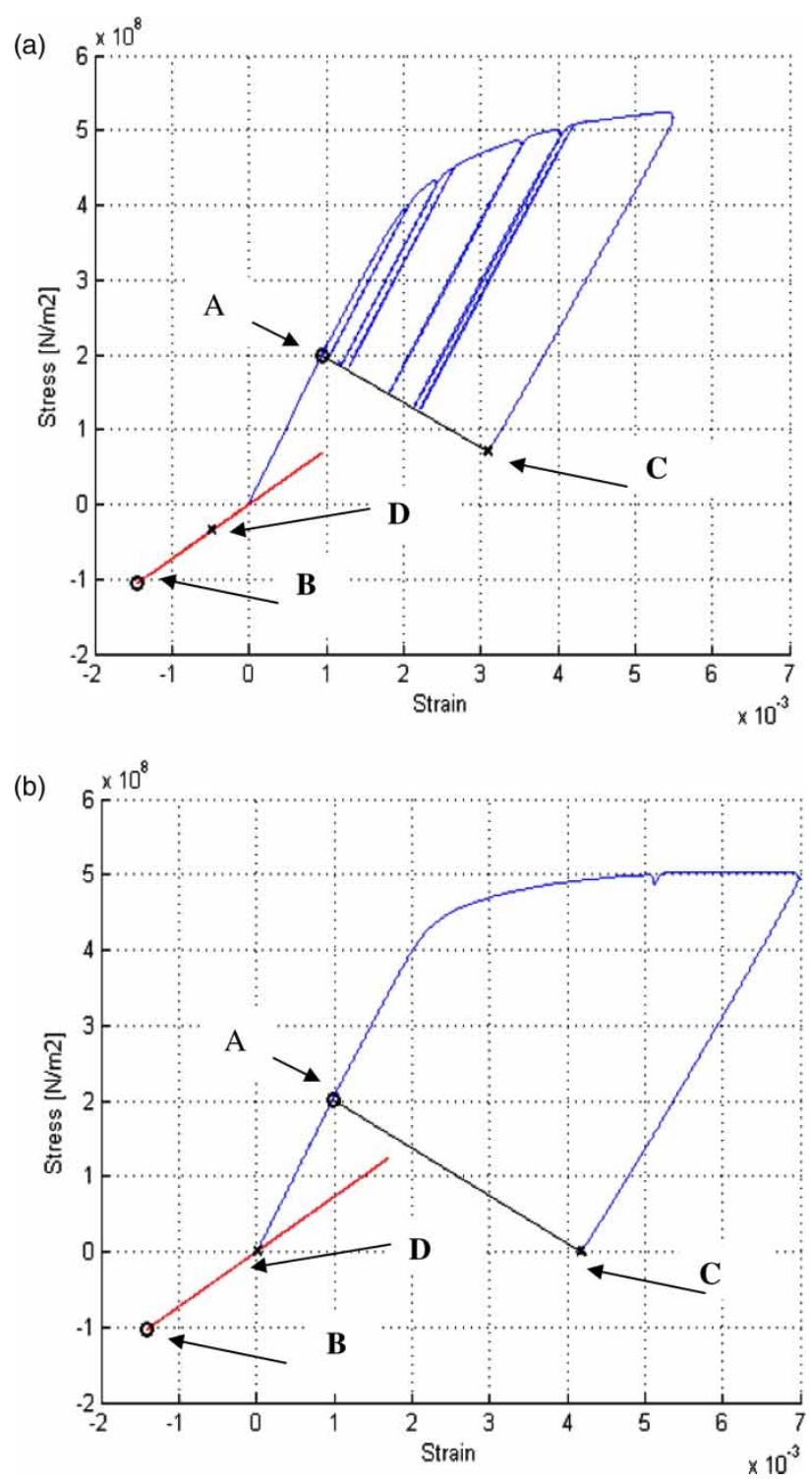

Fig. 8 Stress and strain response of preloaded three-bar system: (a) multiple load cycles and (b) single load cycle. Points A and B are the initial misfit stresses in the central steel bar and in an aluminium outer bar, points $C$ and $D$ are the final misfit stresses in the steel and aluminium bars

the initial stress in the central bar and point B the initial stress in one of the two outer bars. Evidently, the stresses in the assembly sum to zero. The line joining points $\mathrm{A}$ and $\mathrm{C}$ represent the locus of relaxed stresses in the central bar. The final stresses and strains in the assembly corresponding to points $C$ and D are shown in Table 2 along with other parameters associated with the test. Fig. 8(b) shows the results for a second 3-bar assembly where a single load cycle was prescribed rather than subjecting the assembly to multiple load cycles. Removal of the external loading revealed that the initial stresses in the central and outer bars had relaxed to zero because the plastic strain in bar 1 had accommodated the initial misfit.
Table 2 Measured states of the three-bar assembly

\begin{tabular}{lll}
\hline & $\begin{array}{l}\text { Central } \\
\text { steel bar }\end{array}$ & $\begin{array}{l}\text { Outer } \\
\text { aluminium bar }\end{array}$ \\
\hline Elastic modulus (GPa) & 211.7 & 73.7 \\
Initial misfit stress (MPa) & 199.0 & -105.6 \\
Initial misfit strain, microstrain & 940 & -1477 \\
Final misfit stress (MPa) & 70.4 & -35.0 \\
Final misfit strain, microstrain & 3098 & 48 \\
\hline
\end{tabular}

Table 3 Measured states of the seven-bar assembly

\begin{tabular}{lll}
\hline & $\begin{array}{l}\text { Central } \\
\text { steel bar }\end{array}$ & $\begin{array}{l}\text { Outer } \\
\text { aluminium bar }\end{array}$ \\
\hline Elastic modulus (GPa) & 213.7 & 73.7 \\
Initial misfit stress (MPa) & 191.4 & -40.3 \\
Initial misfit strain, microstrain & 908 & -552 \\
Final misfit stress (MPa) & 78.4 & -11.2 \\
Final misfit strain, microstrain & 1847 & 154 \\
\hline
\end{tabular}

Identical calculations were applied to the results from the five- and seven-bar (Table 3) parallel assemblies, each with a standard central steel specimen. The results, shown in Fig. 9, indicate that similar characteristics to those observed in Fig. 8 are evident. An important feature of the behaviour of these assemblies is that the relaxation line $\mathrm{A}-\mathrm{C}$ has a slope dependent on the relative stiffness of the assembly.

\subsection{Experimental determination of elastic follow-up}

Two methods were used to determine $Z$. The first method used equation (3) with the strains $\varepsilon_{\mathrm{f}}, \varepsilon_{\mathrm{e}}$, and $\varepsilon_{\mathrm{i}}$ measured and derived from the experiments. For example, Fig. 10 shows the values of these strains for the three-bar parallel assembly subjected to multiple loadings. While the final $\varepsilon_{\mathrm{f}}$ and equivalent elastic $\varepsilon_{\mathrm{e}}$ strains can be determined directly from diagrams, similar to that shown in Fig. 10, it was required to determine the initial elastic strain from the applied load to each assembly.

As shown in Appendix 1, the total maximum load applied to the assembly assuming a fully elastic system is given by

$$
F_{\mathrm{tm}}=K_{\mathrm{S}} \Delta_{\mathrm{m}}+n K_{\mathrm{A}} \Delta_{\mathrm{m}}
$$

where $K_{\mathrm{S}}$ is the stiffness of the central steel bar and $\Delta_{\mathrm{m}}$ is the maximum applied displacement of the assembly corresponding to the maximum applied force $F_{\mathrm{tm}}$.

Rearranging equation (14) gives

$$
\Delta_{\mathrm{m}}=\frac{F_{\mathrm{tm}}}{K_{\mathrm{S}}+n K_{\mathrm{A}}}
$$

Therefore, the equivalent elastic strain applied to the central steel bar, including the initial misfit strain $\varepsilon_{0}$, is 

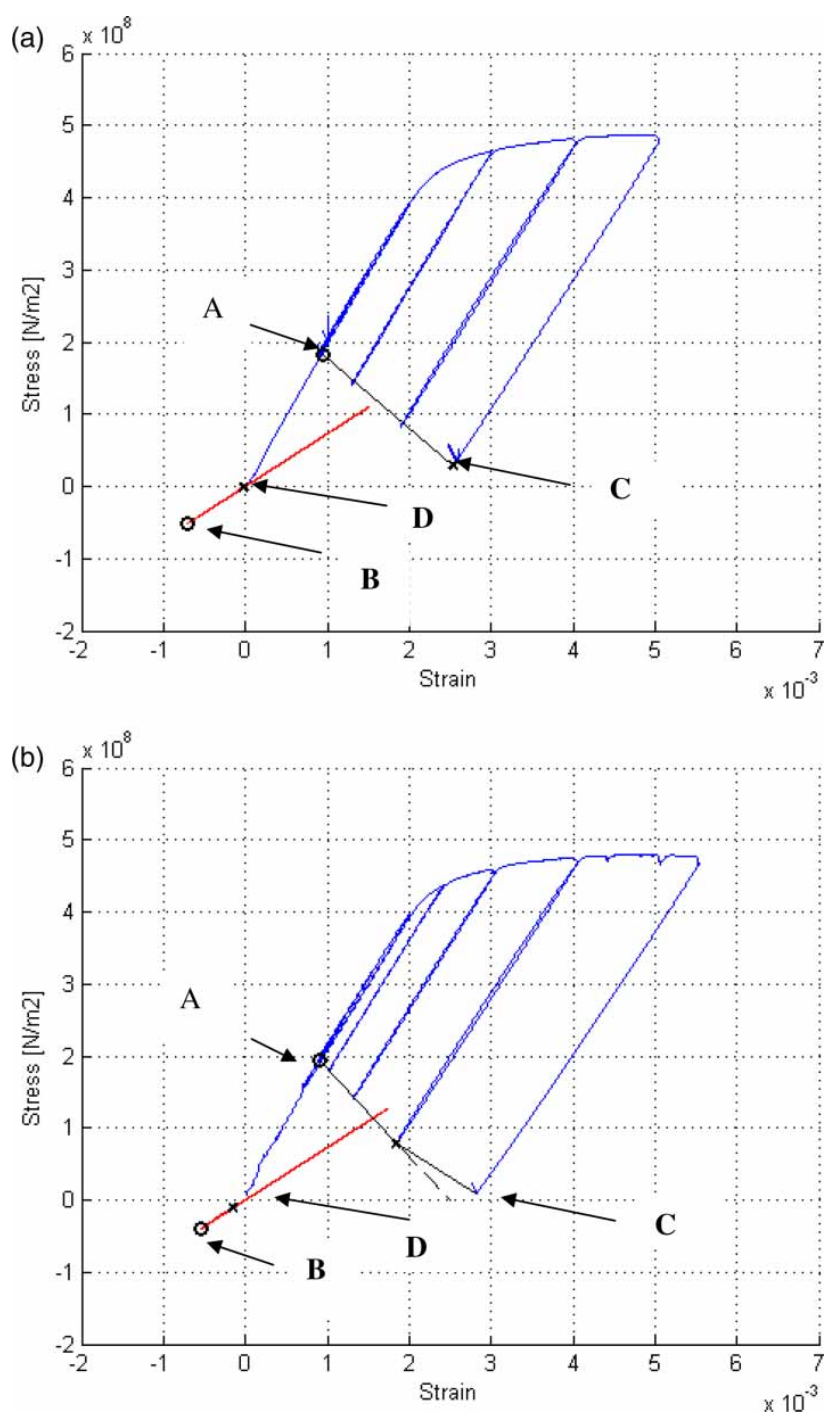

Fig. 9 Stress and strain response of a preloaded multiple bar system subjected to multiple loading cycles: (a) five-bar system; (b) seven-bar system. Points $A$ and $B$ are the initial misfit stresses in the central steel bar and in an aluminium outer bar, points $C$ and $\mathrm{D}$ are the final misfit stresses in the steel and aluminium bars

given by

$$
\varepsilon_{\mathrm{i}}=\frac{\Delta_{\mathrm{m}}}{L_{\mathrm{S}}}+\varepsilon_{0}
$$

The measured values of $Z$ using equation (3) (i.e. method 1) are shown in Table 1 together with the measured values of relative stiffness. The values of $Z$ given in Table 1 correspond to the maximum applied loads prior to unloading.

The second method for determining the elastic follow-up factor uses equation (10) derived from the strains associated with the initial misfit strains and their subsequent relaxation. All the strains in equation (10) were measured directly from the tests. Results for $Z_{R}$ are shown in Table 1.

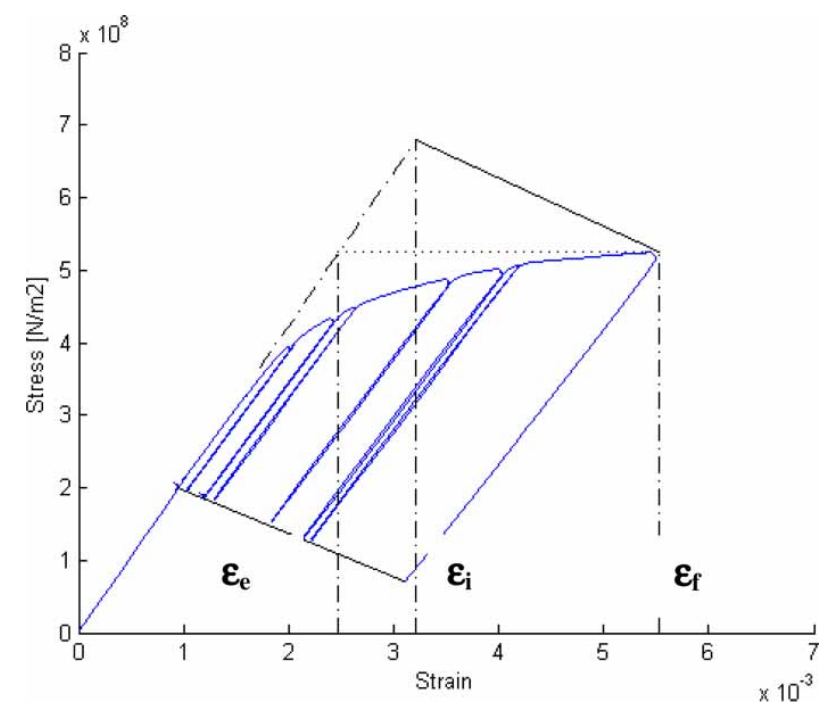

Fig. 10 Measured strains for determination of elastic follow-up factor, $Z$. Illustrated case for three-bar parallel assembly

It is apparent from the results shown in Table 1 that the two methods of determining the elastic follow-up factor from the experiments agree approximately. However, the measured values for the parallel assembly tests are substantially greater than predicted by simple theory. In contrast, the measured values obtained from the combined series and parallel systems were less than predicted from simple theory. The measured values of elastic follow-up factor, derived from the two methods, are also illustrated in Fig. 11. For an assembly that was intended to contain parallel elements alone it is apparent that the experimental results lie on curves that suggest the assembly was a combination of series and parallel elements.

The experimental results for $Z$ and $Z_{\mathrm{R}}$, shown in Table 1 and Fig. 11, correspond to the maximum plastic strain observed in the central bar. However, as the

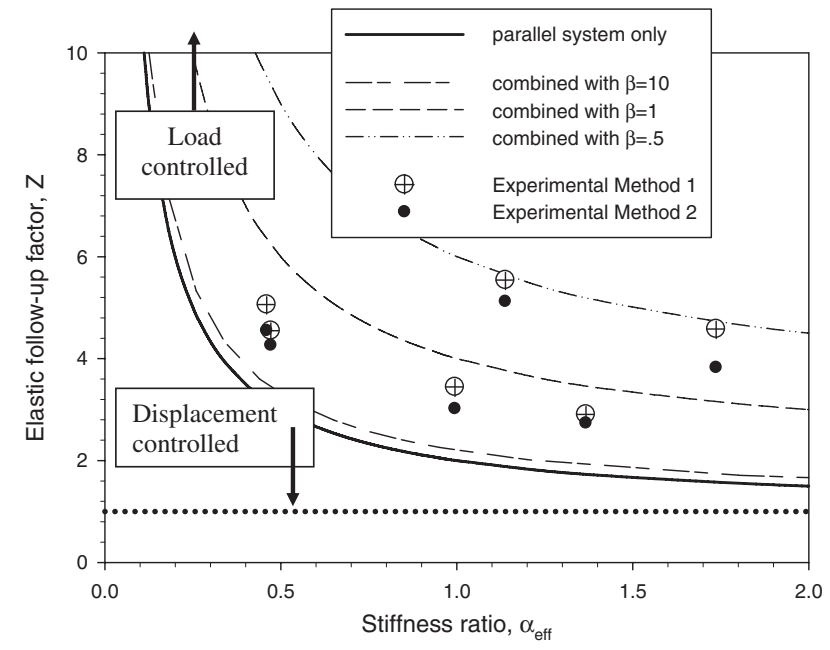

Fig. 11 Comparison of predicted and measured elastic follow-up 


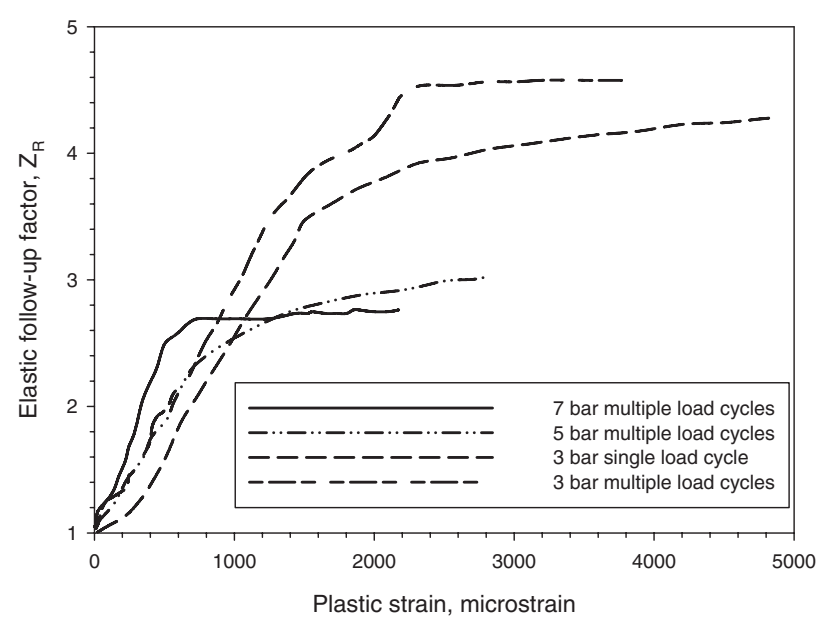

Fig. 12 Change in elastic follow-up factor $Z_{\mathrm{R}}$ with increasing plastic strain for parallel bar assemblies

experimental results illustrate in Fig. 8, there was a degree of strain hardening prior to final unloading. The amount of plastic strain dictated the degree of residual stress relaxation for each assembly and this is shown in Fig. 12 for the three-, five-, and seven-bar assemblies. It is evident that $Z_{\mathrm{R}}$ increases with increasing strain hardening. This is discussed in the next section.

\section{DISCUSSION}

The experiments mimicked the essential features of the structural behaviour of the assemblies predicted by simple theory. Two significant characteristics were manifest: elastic follow-up was dictated by the relative stiffness of the elements of the assembly and equally the rate of relaxation of an initial or residual stress in the central bar was controlled by relative stiffness. In principle, the overriding purpose of quantifying elastic follow-up is to determine the additional strain accumulated as a direct consequence of the other elements in the assembly redistributing the applied stresses. In turn, quantifying the elastic follow-up factor permits an evaluation of how the residual stress relaxes as the initial misfit is accommodated.

The measurements of the elastic follow-up factor for the various parallel assemblies studied in this work tended to provide values greater than predicted. This plainly indicates that more plastic strain was observed in the central bar than predicted by theory. Therefore, the assumed stiffness of the parallel assembly was incorrect. The results illustrated in Fig. 11 also suggest this. The calculated estimates of relative stiffness for the assemblies were based on the main structural elements (i.e. the bars). No account had been taken on features such as blend radii and the presence of the shoulder to connect the central bar to the upper and lower sections. Estimates of their contribution to the stiffness of the assembly did not significantly change the relative stiffness $\alpha_{\text {eff }}$ of the parallel assembly, but introduced an additional series relative stiffness to the central bar, $\beta$. For example, a typical value of $\beta$ equal to $\sim 5$ was found. For the three-bar assembly the predicted value of $Z$ therefore changed from 3 to 3.7, still lower than the measured values of $Z$ and $Z_{\mathrm{R}}$.

The contributions of the additional elements to the stiffness of a combined series and parallel assembly were not as significant, partly because the relative stiffness of the central bar was dominated by the reduced section. Revised values of predicted $Z$ were not that different from those shown in Table 1, with measured values less than predicted. Overall, the experimental results have shown that the most effective method of determining an elastic follow-up factor for each assembly was through the relaxation of the initial stress (i.e. using line AC in Figs 4, 8, and 9). Although there was uncertainty in the determination of the relative stiffness of an assembly, the measurements of residual stress relaxation, as shown in Fig. 11, validated the simple theory.

The original intention of the study was not only to measure and compare elastic follow-up, but also to provide insight into the role played by initial stresses. As expected, their presence automatically changed the conditions for onset of plastic flow. For example, when the residual stress was uniformly tensile, as shown in Figs. 8 and 9, the applied stress to initiate yielding was lower. With continued plastic deformation the assembly readjusts, thereby subjecting the central bar to elastic follow-up. Notably, the presence of residual stresses does not contribute to follow-up. Rather the occurrence of plasticity was a starting point for the relaxation of the initial residual stress. The rate of relaxation of the initial residual stress can be determined by using equation (9). The residual force in the steel central bar can be simply written in terms of stress using equation (13) as

$$
\sigma_{\mathrm{SR}}=E_{\mathrm{S}} \varepsilon_{0}\left(\frac{\alpha_{\mathrm{eff}}}{1+\alpha_{\mathrm{eff}}}\right)
$$

The rate of relaxation of the residual stress, $\sigma_{\mathrm{SR}}$ due to the change in misfit produced by the accumulation of plastic strain in the central bar is then

$$
\frac{\partial \sigma_{\mathrm{SR}}}{\partial \varepsilon_{0}}=E_{\mathrm{S}}\left(\frac{\alpha_{\mathrm{eff}}}{1+\alpha_{\text {eff }}}\right)=\frac{E_{\mathrm{s}}}{Z_{\mathrm{peff}}}
$$

Consequently, as equation (18) reveals, the rate of relaxation of the residual stress is inversely proportional to the elastic follow-up factor for the parallel system. However, the analysis assumed an elasticperfectly-plastic material model for the central bar. As Fig. 12 shows, the measured elastic follow-up factor $Z_{\mathrm{R}}$ was dependent on the plastic strain and strain hardening and suggests that the rate of residual stress relaxation also depends on strain hardening. There is additional indirect evidence provided by Kobayashi et al. 
[15] to demonstrate that strain hardening changes elastic follow-up. They illustrate that the elastic followup strain initially increases with increasing initial strain, similar to the results portrayed in Fig. 12.

\section{CONCLUSIONS}

An experimental study of the structural behaviour of an assembly has confirmed that the predicted features of elastic follow-up and residual stress relaxation are observed. Experiments were undertaken using a variety of parallel and combined series and parallel assemblies that provided different relative stiffness and hence different conditions for elastic follow-up. The study revealed that the most reliable estimate of elastic follow-up was measured via the relaxation of the initial residual stress. There was general agreement between measured and predicted values of the elastic follow-up factors with the greatest uncertainty associated with accurate determination of the stiffness of the assemblies.

It has also been demonstrated that initial residual stresses do not contribute to elastic follow-up; rather their role is to promote or suppress the initiation of plasticity. It is shown that the rate of residual stress relaxation is inversely proportional to the elastic follow-up factor.

\section{ACKNOWLEDGEMENTS}

This work was supported by the MOD NPCT programme. David Smith is also grateful for the support of a Royal Society Wolfson Merit Award.

(C) Authors 2010

\section{REFERENCES}

1 Masubuchi, K. Analysis of welded structures: residual stresses, distortion, and their consequences, 1980 (Pergamon Press, Oxford).

2 Anderson, T. L. Fracture mechanics - fundamentals and applications, 3rd edition, 1995 (CRC Press, Baco Raton).

3 Rees, D.W. A. Autofrettage theory and fatigue life of openended cylinders. J. Strain Analysis, 1990, 25(2), 109-121. DOI: 10.1243/03093247V252109.

4 Lacarac, V. D., Garcia-Granada, A. A., Smith, D. J., and Pavier, M. J. Comparison of predicted and measured growth rates for a fatigue crack emanating from a cold expanded hole. Int. J. Fatigue, 2004, 26, 585-595.

5 Rao, K. R. Companion guide to the ASME boiler \& pressure vessel code, vol. 1, 2002, pp. 194-208 (The American Society of Mechanical Engineers, New York).

6 British Energy. R6, assessment of the integrity of structures containing defects, Revision 4, British Energy Generation Limited, 2001.

7 BSI-British Standards Institute, BS7910. Guide to methods for assessing the acceptability of flaws in metallic structures, 2005 (British Standards Institute (BSI), London).

8 RCC-MR Code. Design and Construction Rules for Mechanical Components of FBR Nuclear Islands and High Temperature Applications, Appendix A16, AFCEN, 2007.

9 Robinson, E. L. The resistance to relaxation of materials at high temperature. Trans. Am. Soc. Mech. Eng., 1939, 61, 543-550.

10 Teramae, T. A simplified method for elastic follow-up analysis of elevated temperature piping systems. Int. J. Press. Vessels Pip., 1983, 12, 29-41.

11 Dhalla, A. K. and Jones, G. L. ASME code classification of pipe stresses: a simplified elastic procedure. Int. J. Press. Vessels Pip., 1986, 26, 145-166.

12 Roche, R. L. Estimation of piping elastic follow-up by using conventional computations. Int. J. Press. Vessels Pip., 1986, 26, 53-78.

13 Boyle, J. T. and Nakamura, K. The assessment of elastic follow-up in high temperature piping systems - overall survey and theoretical aspects. Int. J. Press. Vessels Pip., 1987, 29, 167-194.

14 Kobayashi, K., Abe, S., and Udoguchi, T. Stress and strain behaviors under uniaxial elastic follow-up. Bull. JSME, 1986, 257, 3672-3678.

15 Kobayashi, K., Saitoh, Y., and Udoguchi, T. Estimation of elastic follow-up behavior on $18 \mathrm{Cr}-8 \mathrm{Ni}$ steel using simplified inelastic analysis. ASME, J. Press. Vessel Technol., 1994, 116, 136-140.

16 Kobatake, K., Ohta, H., Ishiyama, H., Kaihara, T., and Ueno, O. Notched bars test of creep-fatigue damage with elastic follow-up for high temperature structural design. J. Natl. Fish. Univ., 2000, 48, 233-244.

17 Seshadri, R. Residual stress estimation and shakedown evaluation using GLOSS analysis. ASME, J. Press. Vessel Technol., 1994, 116, 290-294.

18 Kasahara, N. Strain concentrations at structural discontinuities and its prediction on characteristics of compliance change in structures. JSME Int. J., 2001, 44, 354-361.

19 Kasahara, N., Nagata, T., Iwata, K., and Negishi, H. Advanced creep-fatigue evaluation rule for fast breeder reactor components: generalization of elastic follow-up model. Nucl. Eng. Des., 1995, 15(5), 499-518.

20 British Energy Generation Ltd. R5, Assessment procedure for the high temperature response of structures, Issue 3, 2003.

21 Aird, C., Hadidi-moud, S., Truman, C. E., and Smith, D. J. Impact of residual stress and elastic follow-up on fracture. J. ASTM Int., 2008, 5(8), JAI 101608.

22 British Standard BS EN 20001-1. Metallic materialstensile testing, 2001.

23 Hadidi-Moud, S. and Smith, D. J. Use of elastic follow-up in integrity assessment of structures. In Proceedings of PVP2008, Chicago, Illinois, USA, 27-31 July 2008, paper 61754 .

\section{APPENDIX 1}

\section{Notation}

A cross-sectional area

E Young's modulus

$F \quad$ force on element/bar 
$K \quad$ stiffness of an element/bar

$L \quad$ length of element/bar

$n$ number of elements/bar

$Z \quad$ elastic follow-up factor

$\alpha \quad$ ratio of stiffness of parallel elements

$\beta \quad$ ratio of stiffness of series elements

$\delta \quad$ local displacement on element

$\Delta \quad$ displacement applied to whole assembly

$\varepsilon \quad$ strain

$\sigma \quad$ stress

\section{APPENDIX 2}

\section{Basic equations for a multiple bar assembly}

A three-bar assembly is shown in Fig. 1. The assembly is subjected to remote force $F_{\mathrm{t}}$. The force is distributed between the bars, where

$$
F_{\mathrm{t}}=n K_{3} \Delta+K_{\mathrm{eff}} \Delta
$$

where $\Delta$ is the associated total displacement, $n$ the number of outer bars, $K_{3}$ the stiffness of an outer bar, and $K_{\text {eff }}$ the effective stiffness of the central bar given by equation (8). The central bar consists of two bars, 1 and 2 , in series.

Compatibility of displacements provides

$$
\Delta=\delta_{3}=\delta_{1}+\delta_{2}
$$

where $\delta_{1}, \delta_{2}$, and $\delta_{3}$ are the displacements in bars 1,2 , and 3.

When the stress in the central bar 1 exceeds the yield stress, the elastic follow-up factor $Z$ for the assembly is given by

$$
Z=\frac{\varepsilon_{\mathrm{f}}-\varepsilon_{\mathrm{e}}}{\varepsilon_{\mathrm{i}}-\varepsilon_{\mathrm{e}}}
$$

where $\varepsilon_{\mathrm{f}}$ is the final total strain in bar 1 achieved at the maximum load, $\varepsilon_{\mathrm{e}}$ is the equivalent elastic strain provided by the final stress $\sigma_{\mathrm{f}} . \varepsilon_{\mathrm{i}}$ is the initial elastic strain that the central bar would achieve at the maximum load if it had not exhibited plastic deformation. The initial strain is obtained from $\delta_{1}$. This is determined from equation (20), so that

$$
\varepsilon_{\mathrm{i}}=\frac{\delta_{1}}{L_{1}}=\frac{\left(\delta_{3}-\delta_{2}\right)}{L_{1}}
$$

Noting that from equations (19) and (20)

$$
\delta_{3}=\frac{F_{\mathrm{t}}}{n K_{3}+K_{\mathrm{eff}}}
$$

Since the displacement in bar 2 is the same as the combined bars 1-2 then

$$
\delta_{2}=\frac{K_{\text {eff }}}{K_{2}} \delta_{3}
$$

Consequently, substituting equations (23) and (24) into equation (20) gives the initial strain in bar 1

$$
\varepsilon_{\mathrm{i}}=\frac{\delta_{1}}{L_{1}}=\frac{F_{\mathrm{t}}}{K_{1}}\left(\frac{1}{\alpha_{\text {eff }}+1}\right) / L_{1}
$$

The final strain is the response of the assembly to a given load when bar 1 exhibits perfectly plastic behaviour. Once yielding in bar 1 occurs bar 2 remains at the same displacement as there is no further increase in load to cause further elastic displacement. Rewriting equation (19) as

$$
F_{\mathrm{t}}=n K_{3} \Delta+F_{12}
$$

The force $F_{12}$ is now equal to the force to cause yielding in bar $1, F_{1 Y}$. The displacement $\delta_{3}$ is now given by

$$
\delta_{3}=\frac{F_{\mathrm{t}}-F_{1 \mathrm{Y}}}{n K_{3}}
$$

The displacement in bars $1-2$ is now

$$
\delta_{2}=\frac{F_{1 Y}}{K_{2}}
$$

Substituting equations (27) and (28) into equation (20) and normalizing with respect to the gauge length gives the final strain in bar 1

$$
\varepsilon_{\mathrm{f}}=\frac{\delta_{1}}{L_{1}}=\left[\frac{F_{\mathrm{t}}}{n K_{3}}-F_{1 \mathrm{Y}}\left(\frac{1}{K_{2}}+\frac{1}{n K_{3}}\right)\right] / L_{1}
$$

The elastic strain $\varepsilon_{\mathrm{e}}$ is the elastic response of bar 1 for the same final stress and is given by

$$
\varepsilon_{\mathrm{e}}=\frac{F_{1 \mathrm{Y}}}{K_{1} L_{1}}
$$

By substituting equations (27), (29), and (30) into equation (21), the elastic follow-up factor in bar 1 is

$$
Z=\frac{K_{1}}{K_{\text {eff }}}\left\{\frac{F_{\mathrm{t}}-F_{1 \mathrm{Y}}\left(1+\alpha_{\mathrm{eff}}\right)}{F_{\mathrm{t}}\left[\alpha_{\mathrm{eff}} /\left(1+\alpha_{\mathrm{eff}}\right)\right]-\alpha_{\mathrm{eff}} F_{1 \mathrm{Y}}}\right\}
$$

The term in square brackets can be shown to be equal to $\left(1+\alpha_{\text {eff }}\right) / \alpha_{\text {eff }}$ and

$$
\frac{K_{1}}{K_{\text {eff }}}=\frac{1+\beta}{\beta}
$$

Consequently, $Z$ is given by

$$
Z=\left(\frac{1+\alpha_{\text {eff }}}{\alpha_{\text {eff }}}\right)\left(\frac{1+\beta}{\beta}\right)
$$

Further details for elastic-creep behaviour are considered by Hadidi-moud and Smith [23].

When there are no parallel bars and only a series system, equation (33) reduces to

$$
Z=Z_{\mathrm{s}}=\frac{1+\beta}{\beta}
$$

For a parallel system alone, $\alpha_{\text {eff }}=\alpha$ and

$$
Z=Z_{\mathrm{p}}=\frac{1+\alpha}{\alpha}
$$

